\title{
Multi-phase Three-Dimensional Level Set Segmentation of Brain MRI
}

\author{
Elsa D. Angelini ${ }^{1}$, Ting Song ${ }^{2}$, Brett D. Mensh ${ }^{3}$, and Andrew Laine ${ }^{2}$ \\ 'Ecole Nationale Supérieure des Télécommunications, Département Traitement du Signal et \\ des Images, 46 rue Barrault, 75013 Paris, France. \\ elsa.angeliniaenst.fr \\ ${ }^{2}$ Columbia University, Heffner Biomedical Imaging Laboratory, \\ Department of Biomedical Engineering, 1210 Amsterdam Avenue, \\ New York, NY 10027, USA. \\ \{ts2060, laine\} @columbia. edu \\ ${ }^{3}$ Columbia University, Department of Biological Phsychiatry, College of Physicians and \\ Surgeons, Columbia University, New York, NY, 10032, USA. \\ bm189@columbia.edu
}

\begin{abstract}
This paper presents the implementation and quantitative evaluation of a four-phase three-dimensional active contour implemented with a level set framework for automated segmentation of cortical structures on brain T1 MRI images. The segmentation algorithm performed an optimal partitioning of three-dimensional data based on homogeneity measures that naturally evolves to the extraction of different tissue types in the brain. Random seed initialization was used to speed up numerical computation and avoid the need for $a$ priori information. A simple post-processing, based on morphological operators, was applied to correct for segmentation artifacts. The segmentation method was tested on ten MRI brain data sets and quantitative evaluation was performed by comparison to manually labeled data, Computation of false positive and false negative assignments of voxels for white matter, gray matter and cerebrospinal fluid were performed. Results reported high accuracy of the segmentation methods, demonstrating the efficiency and flexibility of the multi-phase level set segmentation framework to perform the challenging task of automatically extracting cortical brain tissue volume contours.
\end{abstract}

\section{Introduction}

This paper presents the implementation and quantitative evaluation of a four-phase three-dimensional active contour implemented within a level set framework for automated segmentation of cortical brain structure on $\mathrm{T} 1$ magnetic resonance images (MRI). A level set implementation of surface propagation offers the advantage of easy initialization, computational efficiency, and the ability to capture deep sulcal folds. In recent years, several works have focused on using level set methods for MRI brain segmentation. Zeng et al. [1] proposed a segmentation method of the cortex from 3-D MR images using coupled level set surface propagation, assuming a constant thickness range of the cortical mantle. By evolving two embedded surfaces simultaneously, each 
driven by its own image-derived information and within a certain distance range from each other, a segmentation of the cortical gray matter was achieved. Recently, Yang et al. [2] proposed a new method for three-dimensional MRI segmentation based on the combination of joint-prior shape appearance models within a level set deformable model. This method was motivated by the observation that the shapes and gray levels variations in an image had some consistent relations. Building a maximum $a$ posteriori shape-appearance prior model provided some configurations and context information to assist the segmentation process. The model was formulated in a level set framework rather than using landmark points for parametric shape description. Goldenerg et al.[3] formulated their 3D MR image segmentation problem as a geometric variational problem for propagation of two coupled bounding surfaces, similar to Zeng et al.[1] . The authors put forward an efficient numerical scheme for the implementation of a geodesic active surface model, where a surface evolution was performed.

In this work, we have implemented the multi-phase level set framework first proposed by Chan and Vese [4]. This framework simultaneously deforms coupled level set functions without any prior models or shape constraints. A global partition of the image data results in $2^{\mathrm{N}}$ homogenous areas for $N$ level set curves, solely based on average gray values measures.

\section{Method}

\subsection{Active Contours Without Edges}

A new energy functional for homogeneity-based segmentation derived from the work of Mumford and Shah was proposed by Chan and Vese [5]. Let us assume that a given image, $u_{0}$ is formed by two regions of approximate piecewise constant intensities, of distinct values $u_{0}^{i}$ and $u_{0}^{o}$. Let us denote the boundary between the two regions by $C_{0}$. Given an initial curve $C$ defined on the image, the following "fitting energy" can be minimized to segment the two regions:

$$
F_{1}(C)+F_{2}(C)=\int_{\text {inside }(C)}\left|u_{0}-c_{1}\right|^{2} d x+\int_{\text {outside }(C)}\left|u_{0}-c_{2}\right|^{2} d x .
$$

The parameters $c_{1}, c_{2}$ correspond to the mean values of the image inside and outside the curve $C$. The curve $C_{0}$ that corresponds to the boundary of the object minimizes this energy functional:

$$
\inf _{C}\left\{F_{1}(C)+F_{2}(C)\right\}=0=F_{1}\left(C_{0}\right)+F_{2}\left(C_{0}\right)
$$

As a special case of the Mumford-Shah functional, Chan and Vese proposed an active contour model derived from this energy functional with the addition of two regularizing terms to constrain the length of $C$ and the area inside $C$ :

$$
E=\mu(\text { length }(C))+v(\operatorname{area}(\text { inside } C))+\lambda_{1} \int_{\text {inside }(C)}\left|u_{0}-c_{1}\right|^{2} d x+\lambda_{2} \int_{\text {inside }(C)}\left|u_{0}-c_{2}\right|^{2} d x
$$

where $\mu \geq 0, v \geq 0, \lambda_{1}, \lambda_{2}>0$ are fixed parameters. 
This energy functional can be extended to the segmentation of multiple homogeneous objects in the image by using several curves $\left\{C_{1}, C_{2}, \ldots, C_{i}\right\}$. In the case of two curves we use the following fitting energy.

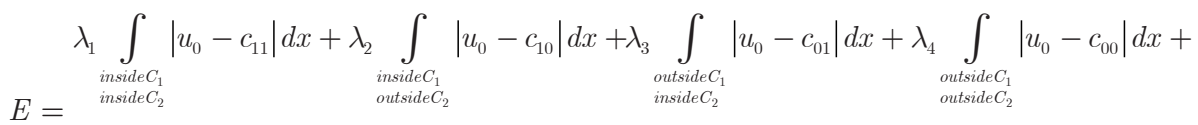

$$
\begin{aligned}
& \mu_{1} \text { length }\left(C_{1}\right)+\mu_{2} \text { length }\left(C_{2}\right)+v_{1} \text { area }\left(\text { inside } C_{1}\right)+v_{2} \text { area }\left(\text { inside } C_{2}\right)
\end{aligned}
$$

Minimization of this energy functional deforms simultaneously two curves and identifies four homogeneous areas defined by the intersection of the two curves as illustrated in Fig. 1.

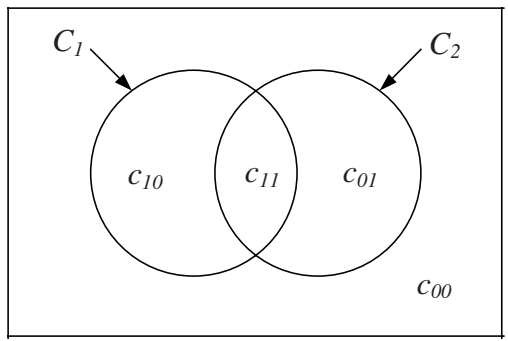

Fig. 1. Partitioning of an image into four areas with two curves $C_{1}, C_{2}$.

\subsection{Level Set Formulations of Minimization Problems}

Minimization of the functional in Equation (3) is performed with a level set implementation. The level set framework, introduced by Osher and Sethian [6], provides an effective implicit representation for evolving curves and surfaces, which has found many applications, as it allows topological changes, such as merging and breaking.

In this framework, a given curve $C$ (being now the boundary of an open set $\omega \in \Omega$ ) is represented implicitly, as the zero level set of a scalar Lipschitz function, called the level set function, negative inside $\omega$, positive outside $\omega$ and zero on the contour. Given the curve $C$ embedded in a level set function $\phi$, its associated Heaviside function $H$ and Dirac function $\delta$ are defined respectively as described in [4]. Using these functions, the different components of the functional in Equation (3), parameterized with the contour curve $C$, can be reformulated with the level function $\phi$ as described in [4].

Detection of multiple objects is performed via the introduction of multiple level set functions $\left\{\phi_{1}, \phi_{2}\right\}$ and the computation of mean data values in areas of constant values is defined via the combination of their Heaviside functions $\left\{H\left(\phi_{1}\right), H\left(\phi_{2}\right)\right\}$. In this study we implemented the segmentation functional with two level set functions generating four phases defined with mean values defined as: 


$$
\begin{gathered}
c_{11}\left(\phi_{1}, \phi_{2}\right)=\frac{\int_{\Omega} u_{0}(x) H\left(\phi_{1}\right) H\left(\phi_{2}\right) d \Omega}{\int_{\Omega} H\left(\phi_{1}\right) H\left(\phi_{2}\right) d \Omega}, c_{10}\left(\phi_{1}, \phi_{2}\right)=\frac{\int_{\Omega} u_{0}(x) H\left(\phi_{1}\right)\left(1-H\left(\phi_{2}\right)\right) d \Omega}{\int_{\Omega} H\left(\phi_{1}\right)\left(1-H\left(\phi_{2}\right)\right) d \Omega},(4) \\
c_{01}\left(\phi_{1}, \phi_{2}\right)=\frac{\int_{\Omega} u_{0}(x)\left(1-H\left(\phi_{1}\right)\right) H\left(\phi_{2}\right) d \Omega}{\int_{\Omega}\left(1-H\left(\phi_{1}\right)\right) H\left(\phi_{2}\right) d \Omega}, c_{00}\left(\phi_{1}, \phi_{2}\right)=\frac{\int_{\Omega} u_{0}(x)\left(1-H\left(\phi_{1}\right)\right)\left(1-H\left(\phi_{2}\right)\right) d \Omega}{\int_{\Omega}\left(1-H\left(\phi_{1}\right)\right)\left(1-H\left(\phi_{2}\right)\right) d \Omega}
\end{gathered}
$$

The Euler-Lagrange systems for the two level set functions are defined as:

$$
\begin{aligned}
& \frac{\partial \phi_{1}}{\partial t}=\delta\left(\phi_{1}\right)\left\{\begin{array}{l}
\mu d i v\left(\frac{\nabla \phi_{1}}{\left|\nabla \phi_{1}\right|}\right)-v+\lambda_{1}\left(u_{0}-c_{11}\right)^{2} H\left(\phi_{2}\right)+\lambda_{2}\left(u_{0}-c_{10}\right)^{2}\left(1-H\left(\phi_{2}\right)\right) \\
-\lambda_{3}\left(u_{0}-c_{01}\right)^{2} H\left(\phi_{2}\right)-\lambda_{4}\left(u_{0}-c_{00}\right)^{2}\left(1-H\left(\phi_{2}\right)\right)
\end{array}\right\} \\
& \frac{\partial \phi_{2}}{\partial t}=\delta\left(\phi_{2}\right)\left\{\begin{array}{l}
\mu d i v\left(\frac{\nabla \phi_{2}}{\left|\nabla \phi_{2}\right|}\right)-v+\lambda_{1}\left(u_{0}-c_{11}\right)^{2} H\left(\phi_{1}\right)-\lambda_{2}\left(u_{0}-c_{10}\right)^{2} H\left(\phi_{1}\right) \\
+\lambda_{3}\left(u_{0}-c_{01}\right)^{2}\left(1-H\left(\phi_{1}\right)\right)-\lambda_{4}\left(u_{0}-c_{00}\right)^{2}\left(1-H\left(\phi_{1}\right)\right)
\end{array}\right\}
\end{aligned}
$$

In our implementation we kept the Heaviside function negative inside the contour. This explains the modification of the signs of the homogeneity terms compared to the original paper by Vese [4]. The level set algorithm was implemented with a semiimplicit scheme proposed by Chan and Vese [5] but extended to three dimensions. This implicit scheme provides unconditional stability for any temporal and spatial discretization parameters.

In our implementation, the segmentation was initialized with two level set functions defined as the distance function from two sets of initial curves. The initial curves were defined as 64 cylinders centered at regularly spaced seed locations across the entire data volume and slightly shifted from each other, as illustrated in Fig. 2.

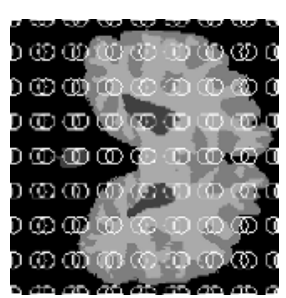

(a)

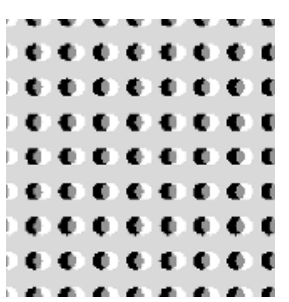

(b)

Fig. 2. Initialization of the level set segmentation. (a) Original MRI slice with two sets of cylinders. (b) Corresponding partitioning of the image domain into 4 phases defined by the overlap of the two level set functions.

Note that such initialization does not use any a priori information on the location of tissues or on the anatomy of the brain and does not require manual input by a user. 


\subsection{Post Processing with Morphological Operators}

Initial experiments on clinical data showed potential misclassification of pixels located at the interface between two structures. This type of error is due to the fact that the segmentation method performs a global segmentation of the data and does not apply any constraints on the size or the shape of the tissues segmented so that misclassification of pixels might cancel each other in the homogeneity measure. To correct for these errors we designed a simple post-processing scheme that adjusted interface pixel assignments. After the level set segmentation was completed, WM, GM and CSF structures, corresponding to separate phases, were saved as binary volumes. These volumes were then used as masks applied to the original data to compute the mean $\mu$ and variance $\sigma$ of the underlying tissue. First, the GM mask was dilated, to correct for any under segmentation of thin structures. According to the statistics of the segmented GM, two threshold values were set as $\mu \pm 3 \sigma$. Contour pixel with gray values inside this interval were kept within the GM phase, while pixels with gray values outside the interval were removed from the phase and assigned to the adjacent WM phase. This process was iterated until no new points were added in the GM phase. A similar process was then applied to the CSF phase with dilation of the binary mask and comparison to the GM with threshold values of $\mu \pm 4 \sigma$ using the CSF statistics.

Finally, a 3-D connectivity algorithm was performed to correct for spurious isolated pixels in the three phases. This simple post-processing approach provided very robust performance on the ten clinical MRI cases segmented in this study, described next.

\section{Experiments and Results}

We applied our segmentation to ten T1-weighted MRI data sets acquired on healthy young volunteers. The MRI data sets were of size $(256 \times 256 \times 73)$ with a $3 \mathrm{~mm}$ slice thickness and $0.86 \mathrm{~mm}$ in-plane resolution. These data sets had been previously labeled via a labor-intensive (40 hours per brain) manual method in which expert raters with extensive training in neuroanatomy choose histogram thresholds on locally handdrawn regions of interest. This labeled data was used as ground truth for evaluation of the segmentation accuracy.

MRI data sets were pre-processed to remove all non-brain tissue by using the corresponding manually labeled data sets as binary masks. Before segmenting the data sets, we evaluated the homogeneity of the three main tissues targeted for segmentation: WM, GM and CSF again using the labeled data for masking each region. Results showed very stable estimates of mean and variance values for each tissue type across the entire volumetric data set, confirming our assumption that a homogeneity-based segmentation method could be applied to these MRI data sets to extract the three main tissue types. A Gaussian fit was performed on the histogram of the entire gray level distribution of the three brain tissues for each case. We observed that the three tissue types have well separated average values suggesting that the assumption of homogeneity and separability of gray scale values is valid for each patient and each tissue type. The agreement between the volume histograms and the fitted Gaussian distribu- 
tion was calculated with a chi-squared test. Results for the different tissues did not show a systematic agreement between the data and the Gaussian fit, at level 0.05, except for the gray matter. Therefore, despite reasonable agreement between the data and the fitted Gaussian distribution, we need further investigation before being able to introduce additional constraints based on a priori Gaussian statistics to the method as proposed for example by Baillard et al.[7] .

As detailed in [8], we used a phantom MRI to tune the parameters of the segmentation method set to:

$$
\begin{aligned}
& \lambda_{1}=\lambda_{2}=\lambda_{3}=\lambda_{4}=0.01, v=0, \quad \mu=4.10^{-8} \times \text { Volume_size } / \text { Diagonal_distance } \\
& \Delta t=10^{4}, \Delta x=\Delta y=\Delta z=1 .
\end{aligned}
$$

The diagonal distance was defined as the diagonal within the data volume. Setting the constant speed term $v$ to zero eliminates the use of a constant inflating force on the model.

\subsection{Quantitative Assessment of Segmentation Performance}

We present in Fig. 3 a selected slice and a three-dimensional rendering of the white matter and CSF cortical structures from a MRI clinical case segmented for this study. Visual rendering of the cortical structures confirmed the overall high performance of the multi-phase segmentation method to extract homogenous objects that correspond to distinct anatomical tissues. The segmentation method was able to handle multiple challenges without any a priori information or shape constraints that include the extraction of highly-convoluted white matter surfaces, the extraction of separate ventricular structures for the CSF, and handling of different volume sizes of the three structures in a simultaneous segmentation scheme.

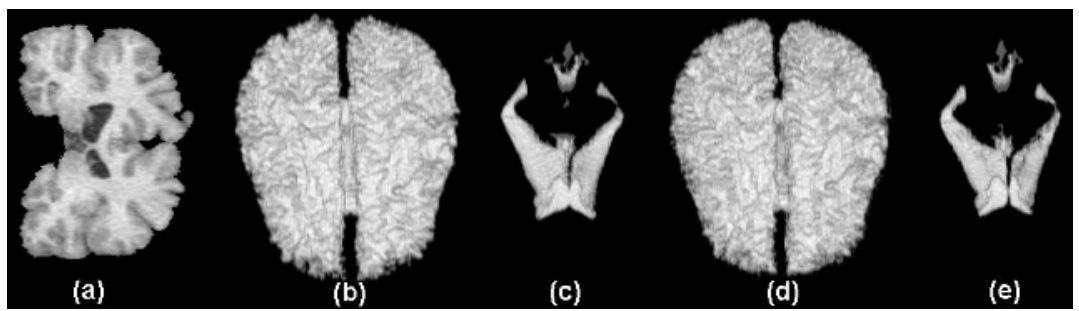

Fig. 3. Rendering of WM and CSF segmented structures. (a) MRI slice. (b-c) Structures from manual labeling. (d-e) Structures from level set segmentation.

Segmentation errors were measured using a recent methodology proposed by Udupa [9] for comparison of segmentation method performance. Accuracy of the object contours obtained with the proposed level set segmentation method was evaluated by comparing the results to our ground truth segmentation of each object, using manually labeled contours. The overlap and difference between the two contours was measured via counting of true positive $(T P)$, false positive $(F P)$ and false negative $(F N)$ voxels. These quantities are reported as volume fractions $(V F)$ of the true delineated object volume in Table 1, for the three clinical cases. 
Table 1. Error measurements for segmentation of clinical MRI cases.

\begin{tabular}{|l|c|c|c|c|c|c|c|c|c|}
\hline \multirow{2}{*}{ Case } & \multicolumn{3}{|c|}{ FNVF (\%) } & \multicolumn{3}{c|}{ FPVF (\%) } & \multicolumn{3}{c|}{ TPVF (\%) } \\
\cline { 2 - 10 } & GM & WM & CSF & GM & WM & CSF & GM & WM & CSF \\
\hline $\mathbf{1}$ & 4.2 & 8.5 & 21.9 & 8.1 & 4.8 & 4.8 & 95.8 & 91.5 & 78.1 \\
\hline $\mathbf{2}$ & 6.5 & 5.1 & 28.5 & 5.0 & 8.9 & 6.1 & 93.5 & 94.9 & 71.5 \\
\hline $\mathbf{3}$ & 4.5 & 6.3 & 27.5 & 5.9 & 5.2 & 7.8 & 95.5 & 93.7 & 72.5 \\
\hline $\mathbf{4}$ & 8.6 & 4.1 & 32.7 & 4.5 & 9.2 & 16.2 & 91.3 & 95.9 & 63.7 \\
\hline $\mathbf{5}$ & 5.4 & 4.3 & 35.7 & 3.9 & 6.8 & 1.0 & 94.6 & 95.7 & 64.3 \\
\hline $\mathbf{6}$ & 5.5 & 18.9 & 26.5 & 17.3 & 0.6 & 0.5 & 99.5 & 81.1 & 73.5 \\
\hline $\mathbf{7}$ & 6.9 & 4.1 & 20.6 & 4.0 & 7.9 & 11.3 & 93.1 & 95.9 & 79.4 \\
\hline $\mathbf{8}$ & 8.3 & 4.6 & 33.8 & 6.4 & 9.3 & 0.005 & 91.7 & 95.4 & 66.2 \\
\hline $\mathbf{9}$ & 8.9 & 2.9 & 37.3 & 2.9 & 11.2 & 0.7 & 91.1 & 97.1 & 62.7 \\
\hline $\mathbf{1 0}$ & 11.0 & 3.8 & 48.7 & 3.9 & 13.5 & 5.3 & 89.0 & 96.2 & 51.3 \\
\hline Average & 7.0 & 6.3 & 31.3 & 6.2 & 7.7 & 5.4 & 93.5 & 93.7 & 68.3 \\
\hline
\end{tabular}

Significantly high FNVF errors were observed for the CSF, corresponding to undersegmentation of the ventricles, whose pixels were assigned to white matter. Very low resolution at the ventricle borders can explain in part this result. On the other hand, labeling of the MRI data for the ventricle can also bear some error as localizations of its borders is difficult even for an expert performing manual tracing. Indeed, Kikinis $e t$ a.l [10] reported a variation in volumetric measurements of manual observers in the order of $15 \%$ for WM, GM and CSF.

We also compared our error measurements to results reported by Zeng et al.[1] and Niessen et al.[11]. In Zeng et al.[1], the authors tested their algorithm for the segmentation of frontal lobes on seven high-resolution MRI datasets from a randomly chosen subset of young autistic and control adult subjects. They ran a coupledsurfaces level set algorithm to isolate the brain tissue and segment the cortex. The average TP and FP volume fractions for the cortical gray matter in the frontal lobe were $86.7 \%$ and $20.8 \%$ (compared to $93.5 \%$ and $6.2 \%$ obtained using the present methods on whole brain). In Niessen et al.[11], a 'hyperstack' segmentation method, based on multiscale pixel classification, was tested for 3D brain MRI segmentation. A supervised segmentation framework with manual post-editing was applied to a probabilistic brain phantom for estimation of segmentation error. First, a binary segmentation of the brain phantom was performed to evaluate the minimal segmentation error due to partial volume effects. The study reported a volume fraction of misclassified pixels (FP+FN) around 20\% for WM, GM and CSF. 'Hyperstack' segmentation was applied with and without a probabilistic framework. Optimal $(\mathrm{FP}+\mathrm{FN})$ volume fraction errors were obtained with the probabilistic version reporting: $10 \%$ for WM, $21 \%$ for GM, and $25 \%$ for CSF.

\section{Conclusion}

This paper presented a novel clinical application and quantitative evaluation of a recently introduced multiphase level-set segmentation algorithm using T1-weighted 
brain MRIs. The segmentation algorithm performed an optimal partitioning of a threedimensional data set based on homogeneity measures that naturally evolves to the extraction of different tissue types in the brain. Experimental studies including ten MRI brain data sets showed that the optimal partitioning successfully identified regions that accurately matched WM, GM and CSF areas. This suggests that by combining the segmentation results with fiducial anatomical seed points, the method could accurately extract individual tissue types from these tissues. Random seed initialization was used to speed up the numerical calculation and avoid convergence to local minima. This random initialization also ensured robustness of the method to variation of user expertise, biased or erroneous a priori input information, and initial settings influenced by variation in image quality. Future work will include incorporation of available co-registered FLAIR and T2-weighted MRI data to improve the segmentation performance for the CSF, running the algorithm on vectorial-type data.

\section{References}

[1] X. Zeng, L. H. Staib, R. T. Schultz, and J. S. Duncan, "Segmentation and measurement of the cortex from 3-D MR images using coupled-surfaces propagation," IEEE Transactions on Medical Imaging, vol. 18, pp. 927-937, 1999.

[2] J. Yang and J. S. Duncan, "3D Image segmentation of deformable objects with shapeappearance joint prior models," MICCAI, Montreal, Canada, pp. 573-580, 2003.

[3] R. Goldenberg, R. Kimmel, E. Rivlin, and M. Rudzsky, "Cortex segmentation - a fast variational geometric approach," IEEE Workshop on Variational and Level Set Methods in Computer Vision, pp. 127-133, 2001.

[4] L. A. Vese and T. F. Chan, "A multiphase level set framework for image segmentation using the Mumford and Shah model," University of California, Los Angeles, CA, USA, Computational and Applied Mathematics Report 01-25, 2001.

[5] T. F. Chan and L. A. Vese, "Active contours without edges," IEEE Transactions on Image Processing, vol. 10, pp. 266 - 277, 2001.

[6] S. Osher and J. A. Sethian, "Fronts propagating with curvature-dependent speed: algorithms based on Hamilton-Jacobi formulation.," Journal of computational physics, vol. 79, pp. 12-49, 1988.

[7] C. Baillard, C. Barillot, and P. Bouthemy, "Robust Adaptive Segmentation of 3D Medical Images with Level Sets," INRIA, Rennes, France, Research Report Nov. 2000.

[8] E. Angelini, T. Song, B. D. Mensh, and A. Laine, "Segmentation and quantitative evaluation of brain MRI data with a multiphase three-dimensional implicit deformable model," SPIE International Symposium Medical Imaging, San Diego, CA, USA, 2004.

[9] J. Udupa, V. LeBlanc, H. Schmidt, C. Imielinska, P. Saha, G. Grevera, Y. Zhuge, P. Molholt, Y. Jin, and L. Currie, "A methodology for evaluating image segmentation algorithm," SPIE Conference on Medical Imaging, San Diego CA, USA, pp. 266-277, 2002.

[10] R. Kikinis, M. E. Shenton, G. Gerig, J. Martin, M. Anderson, D. Metcalf, C. R. Guttmann, R. W. McCarley, W. Lorensen, H. Cline, and F. A. Jolesz, "Routine quantitative analysis of brain and cerebrospinal fluid spaces with MR imaging," Journal of Magnetic Resonance Imaging, vol. 2, pp. 619-629, 1992.

[11] W. J. Niessen, K. L. Vincken, J. Weickert, and M. A. Viergever, "Three-dimensional MR brain segmentation," International Conference on Computer Vision, pp. 53-58, 1998. 
[12] B. Fischl, D. H. Salat, E. Busa, M. Albert, M. Dieterich, C. Haselgrove, A. v. d. Kouwe, R. Killiany, D. Kennedy, S. Klaveness, A. Montillo, N. Makris, B. Rosen, and A. M. Dale, "Whole brain segmentation: automated labeling of neuroanatomical structures in the human brain," Neuron, vol. 33, pp. 341-355, 2002.

[13] W. A. Barrett, L. J. Reese, and E. N. Mortensen, "Intelligent segmentation tools," IEEE International Symposium on Biomedical Imaging, 2002. Proceedings, Washington D.C., USA2002. 